POS PROCEEDINGS

\title{
Construction and performance tests of the COMET CDC
}

\author{
Manabu Moritsu* \\ Institute of Particle and Nuclear Studies (IPNS), High Energy Accelerator Research \\ Organization (KEK) \\ E-mail: moritsuepost.kek.jp
}

Yoshitaka Kuno, Yugo Matsuda, Yu Nakazawa, Kaori Okinaka, Hideyuki Sakamoto, Akira Sato, Ming Liang Wong $\ddagger$ TingSam Wong, Chen Wu, Takahito Yamane, Hisataka Yoshida

Department of Physics, Osaka University

\section{Hai-Bo Li, Jiang Xiaoshan, Jie Zhang}

Institute of High Energy Physics (IHEP)

\begin{abstract}
A cylindrical drift chamber (CDC) has been constructed for the COMET Phase-I experiment where a lepton flavor violating process, the muon-to-electron conversion, is searched for with a sensitivity of $3 \times 10^{-15}$ at J-PARC. The CDC design was optimized to measure $105-\mathrm{MeV} / c$ electrons as well as suppressing unwanted low-energy particles. In order to achieve required momentum resolution of $200 \mathrm{keV} / c$, the chamber is operated with a low- $Z$ gas mixture of He $(90 \%)$ and $i-\mathrm{C}_{4} \mathrm{H}_{10}(10 \%)$ to mitigate the multiple scattering effect. An alternated all stereo layer configuration is also adopted to enhance a good position resolution in the longitudinal direction. The 4,986 sense and 14,562 field wires were strung using gold-plated tungsten wires and unplated aluminum wires, respectively. Proper tensions were applied to control the wire gravitational sag within $55 \pm 7 \mu \mathrm{m}$ for the sense wires, and the sag differences between a given sense wire and surrounding field wires within less than $100 \mu \mathrm{m}$. CDC performance tests using cosmic rays are successfully in progress. We have confirmed decent hit efficiency as well as spatial resolution of $170 \mu \mathrm{m}$ including a tracking uncertainty.
\end{abstract}

The 39th International Conference on High Energy Physics (ICHEP2018)

4-11 July, 2018

Seoul, Korea

\footnotetext{
*Speaker.

$\dagger$ Present address: Center for Computational Science, RIKEN.

${ }^{\ddagger}$ Present address: Université Clermont Auvergne.
} 


\section{Introduction}

The COMET experiment at J-PARC searches for the neutrinoless coherent transition of a muon to an electron in the field of an aluminum nucleus, which violates the lepton flavor conservation and has never been observed yet so far [四, ㅁ] ]. While the process is extremely suppressed in the Standard Model, the conversion rate is predicted to be enhanced in new physics models beyond the Standard Model. The goal of the COMET Phase-I is to explore the muon-to-electron conversion with a single event sensitivity of $3 \times 10^{-15}$, which is 100 times better than the current limit.

In the COMET Phase-I, the converted electrons, which possess monochromatic momentum of $105 \mathrm{MeV} / c$, are detected with a cylindrical drift chamber (CDC) and trigger hodoscopes in a solenoidal magnetic field of $1 \mathrm{~T}$. Figure $\mathbb{W}$ shows a schematic layout of the cylindrical detector system. An inevitable physical background is the decay-in-orbit (DIO) electrons emitted from the normal 3-body muon decay in an atomic orbit. The momentum distribution of the DIO electrons has a high-momentum tail which is able to reach nearly $105 \mathrm{MeV} / c$. In order to distinguish the signal from the DIO background, good momentum resolution of $200 \mathrm{keV} / \mathrm{c}$ is required. Therefore the CDC is designed to reduce material thickness to suppress the multiple scattering effect which dominates momentum resolution in this low energy region. Since low- $Z$ materials have a small photoelectric cross section, they have an additional advantage to reduce background hits caused by low-energy photons from a muon stopping target. Hence we adopt a gas mixture of He: $i-\mathrm{C}_{4} \mathrm{H}_{10}$ (90:10).

The CDC structure consists of inner and outer cylinders with 496 and $835-\mathrm{mm}$ radii and 0.5 and 5-mm thicknesses, respectively, which are made of carbon-fiber-reinforced plastic, as well as aluminum endplates with $1.5-\mathrm{m}$ distance. Owing to the large inner radius, the CDC momentum acceptance opens from $60 \mathrm{MeV} / c$, so that most of low-energy DIO electrons do not reach CDC and are swept away. The cell geometry is almost square shape with $16.8-\mathrm{mm}$ width and $16.0-\mathrm{mm}$

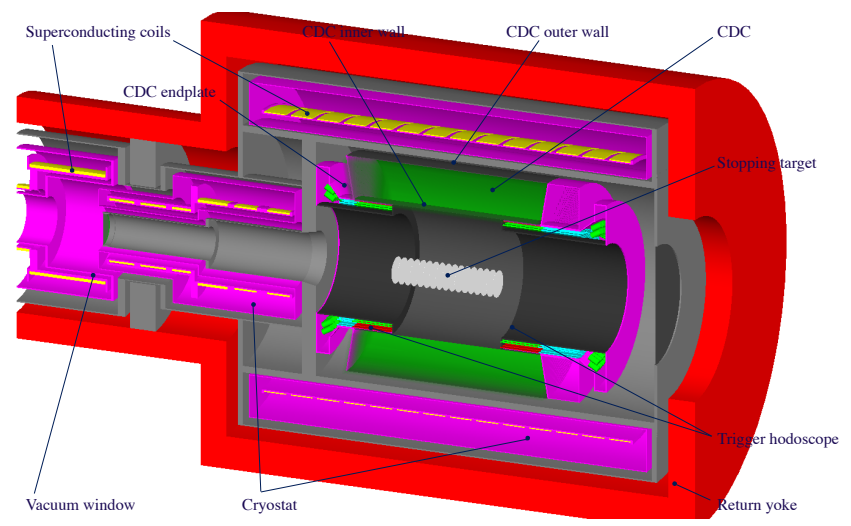

Figure 1: Schematic layout of the cylindrical detector system for the COMET Phase-I. Muon beams come from the left side.

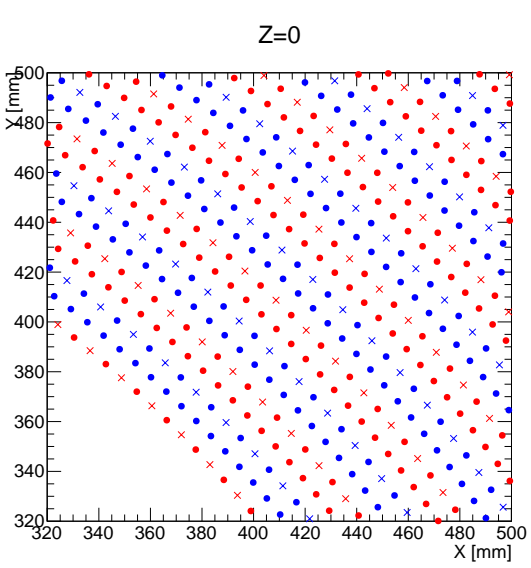

Figure 2: A partial cross section of the CDC cell structure. The crosses and circles indicate sense and field wires. The red and blue colors represent positive and negative stereo angles. 
height. An alternated all stereo layer configuration with $\pm(64-75)$-mrad tilt angles for 20 layers in total is designed to achieve good spatial resolution for the longitudinal direction. As shown in Figure 凹, the cell shapes are slightly distorted from a square according to their positions. This affects an electric field distribution in cell-edge regions, which is taken into account in analyses.

\section{Construction of the Cylindrical Drift Chamber}

The CDC construction was conducted from 2014 to 2016. To handle a endplate distortion by total wire tension of 1.4 ton, the endplates were machined with a step structure which realizes a taper angle of $10^{\circ}$. Wire feedthrough holes were precisely drilled on the endplates with tolerances of 0.01 and $0.05 \mathrm{~mm}$ for the diameter and the position, respectively.

Wire stringing for 19,548 wires in total was performed after attaching the endplates with the outer cylinder. We use gold-plated tungsten wires with $25-\mu \mathrm{m}$ diameter and unplated aluminum wires with 126- $\mu \mathrm{m}$ diameter for the sense and field wires, respectively. To compensate an estimated total wire tension, a pre-stress to the endplates were applied in advance and released appropriately as the wires were strung from outside to inside. The wire tension was measured by a conventional resonance frequency method using alternating current and a magnetic field. The nominal tension weights for the sense and field wires were set to be 50 and $80 \mathrm{~g}$ which correspond to gravitational sags of 50 and $120 \mu \mathrm{m}$, respectively. The gravitational sag at the wire center is given by $\rho L^{2} / 8 w$, where $\rho, L$, and $w$ are the wire linear density, length, and tension weight [B]. Figure B](a) shows the measured sags for the sense wires, which are satisfied between 48 and $61 \mu \mathrm{m}$. Figure B)(b) shows the calculated sag differences between a given sense wire and surrounding field wires. We considered 5 patterns of field-wire relative positions from the given sense wire, which have the same stereo angles. The zigzag structure in the figure reflects the pre-stress release timings. Nevertheless all the sag differences are kept within less than $100 \mu \mathrm{m}$.

The inner cylinder was installed after the wire stringing to close the gas volume of 2,084 litters. The gas tightness was checked with a helium gas and leak points were sealed with silicone

(a) Sag for sense wires

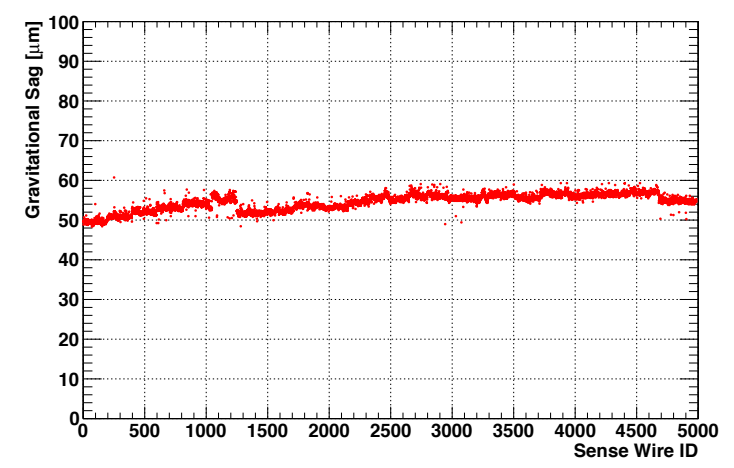

(b) Sag differences between a sense wire and surrounding field wires

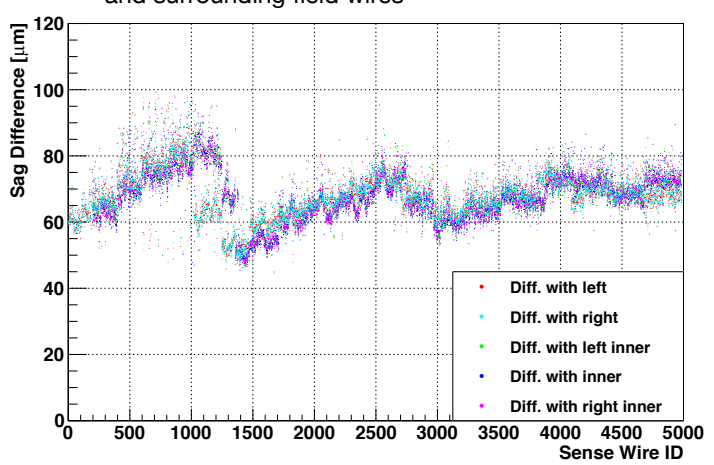

Figure 3: (a) Gravitational sag for sense wires. The horizontal axis denotes a sense wire ID labeled from inside to outside. (b) Gravitational sag differences between a sense and surrounding field wires. The marker colors represent field-wire relative positions from the sense wire. 
gum. We confirmed the gas leakage was less than $20 \mathrm{cc} / \mathrm{min}$, which corresponds to only $1 \%$ of a circulation flow rate of our gas system.

\section{Performance tests of the Cylindrical Drift Chamber}

CDC performance tests using cosmic rays are being carried out. Stable operation of the CDC was established with $\mathrm{He}: i-\mathrm{C}_{4} \mathrm{H}_{10}(90: 10)$ gas mixture and with applied high voltages up to 1850 $\mathrm{V}$. We use readout frontend electronics boards developed for the Belle-II CDC, which provide digitized $960-\mathrm{MHz}$ timing and 30-MHz pulse shape information [䧃]. Figure 因(a) shows a typical event display where a clear cosmic-ray track can be drawn. Figure $\mathrm{G(b)}$ shows a residual distribution obtained from the deviation of drift distance from the distance of closest approach between a hit wire and a reconstructed track. A position resolution is derived to be $170 \mu \mathrm{m}$ including a tracking uncertainty. The right-side tail in the residual distribution appears near the sense wire region due to relatively small numbers of primary electron-ion pairs generated in a cell, which is an intrinsic property of helium-based gas mixtures. Hit efficiency is defined as a fraction of events included in residual within $\pm 3 \sigma$ to total reconstructed tracks. We have obtained a preliminary efficiency of higher than $95 \%$ at $1850 \mathrm{~V}$, and found it was higher than $98 \%$ if we included the right-side tail contribution. The first demonstration of the CDC cosmic-ray tests shows a decent performance as expected, and detailed investigation with large statistics data is in progress.

(a) Event Display

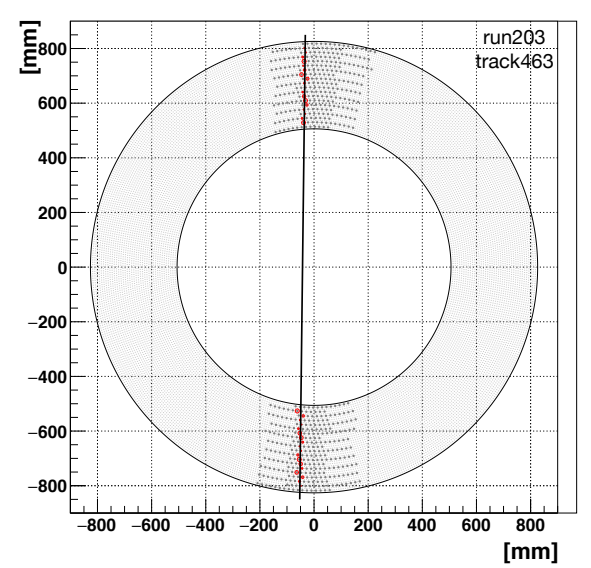

(b) Residual Distribution

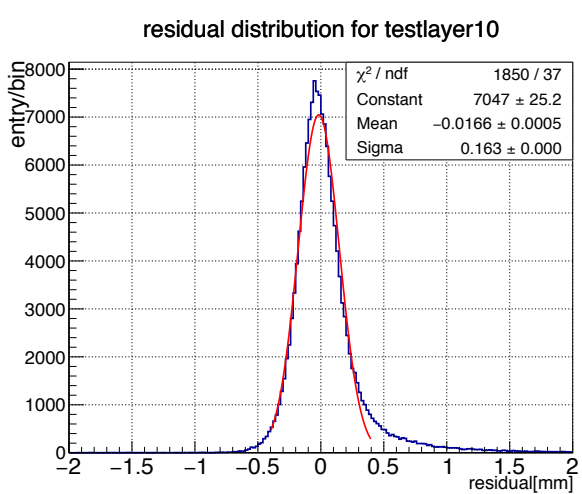

Figure 4: (a) Typical event display in cosmic-ray tests. (b) Residual distribution for a central layer at 1825 V.

\section{References}

[1] http://comet.kek.jp

[2] Y. Kuno (COMET Collaboration), Prog. Theor. Exp. Phys. 2013, $022 \mathrm{C01}$ (2013).

[3] W. Blum, W. Riegler and L. Rolandi, Particle Detection with Drift Chambers, Springer-Verlag 2008.

[4] N. Taniguchi et al., Nucl. Instrum. and Methods A 732, 540 (2013). 